\title{
Twist transition of nematic hyperbolic hedgehogs
}

\author{
Richard James ${ }^{1,2, *}$ and Jun-ichi Fukuda (福田順一) $)^{1, \dagger}$ \\ ${ }^{1}$ Nanosystem Research Institute, National Institute of Advanced Industrial Science and Technology (AIST), 1-1-1 Umezono, \\ Tsukuba 305-8568, Japan \\ ${ }^{2}$ Department of Electronic \& Electrical Engineering, University College London, Torrington Place, London WC1E 7JE, United Kingdom
}

(Received 9 February 2013; revised manuscript received 28 December 2013; published 7 April 2014)

\begin{abstract}
Stability of an idealized hyperbolic hedgehog in a nematic liquid crystal against a twist transition is investigated by extending the methodology of Rüdinger and Stark [Liq. Cryst. 26, 753 (1999)], where the hedgehog is confined between two concentric spheres. In the ideal hyperbolic-hedgehog the molecular orientation is assumed to rotate proportionally with respect to the inclination angle, $\theta$ (and in the opposite sense). However, when splay, $k_{11}$, and bend, $k_{33}$, moduli differ this proportionality is lost and the liquid crystal deforms relative to the ideal with bend and splay. Although slight, these deformations are shown to significantly shift the transition if $k_{11} / k_{33}$ is small. By increasing the degree of confinement the twist transition can be inhibited, a characteristic both hyperbolic and radial hedgehogs have in common. The twist transition of a hyperbolic defect that accompanies a particle is found to be well predicted by the earlier stability analysis of a thick shell.
\end{abstract}

DOI: 10.1103/PhysRevE.89.042501

PACS number(s): 61.30.Cz, 61.30.Jf, 82.20.Wt

\section{INTRODUCTION}

Liquid crystals were first identified by their striking optical textures due to the presence of topological defects [1-4]. In the nematic phase, molecules collectively possess orientational order but lack positional order. Locally, molecules align on average in a direction defined by a unit vector $\hat{n}$, known as the director, which possesses head-tail symmetry such that $\hat{n}$ is equivalent to $-\hat{n}$. Points and lines can arise, termed defects, where the director is ill-defined and correspondingly the degree of orientational order drops.

Stable point defects, those of which are observed in experiment [5], have a topological charge of one and are the radial hedgehog, of charge +1 , and the hyperbolic hedgehog, of charge -1 . Topologically equivalent, one type of hedgehog can be transformed into the other via a twist deformation [6]. Detailed studies of both the radial hedgehog [7-10] and the hyperbolic hedgehog $[11,12]$ reveal that a ring defect of half charge is normally favored at the core $[13,14]$. Only in highly confined systems or at high temperatures is the core restricted to a point [7]. Hedgehogs are relatively rare within the liquid crystal bulk after a quench [5,15], but are commonplace in specific geometries such as capillaries [16-19], droplets [6-8,20], and in liquid crystal colloids [11,21].

Liquid crystals exhibit anisotropy in their elastic moduli, $k_{11}, k_{22}$, and $k_{33}$, respectively, the splay, twist, and bend moduli. Theoretically, and for small deviations in the elastic moduli from the isotropic case, idealized hedgehogs are good representations of the true director configuration and a basis from which the core structure, namely the ring radius, can be calculated in both radial [8,9] and hyperbolic [12] cases as functions of the elastic moduli.

\footnotetext{
*rwjames@gmail.com

†fukuda.jun-ichi@aist.go.jp
}

Published by the American Physical Society under the terms of the Creative Commons Attribution 3.0 License. Further distribution of this work must maintain attribution to the author(s) and the published article's title, journal citation, and DOI.
Relative to the splay and bend moduli, $k_{22}$ is small. Therefore, it can be energetically favorable for a hedgehog defect to twist, breaking mirror symmetry.

For radial hedgehogs the twist transition has been observed experimentally in nematic droplets with homeotropic alignment $[6,20,22]$. Lavrentovich and Terentjev [6] derived a relationship between the elastic moduli at the twist transition by constructing an ansatz connecting a central hyperbolic hedgehog to a radial configuration at the boundary of a droplet via a twist deformation. With this approach, large deformations of the director field are permitted, at the expense of fixing an ansatz function. Rüdinger and Stark take an alternative approach [20]. A linear stability analysis is performed whereby the optimal director configuration is found, but which is valid only for small twist deformations in the vicinity of the transition.

In the case of the hyperbolic hedgehog, the twist transition of the hyperbolic point defect associated with a spherical particle has been studied by Stark [23]. In addition to defining the twist threshold in terms of the elastic moduli, the defect position is reported. Since therein a point defect was simulated by means of the Oseen-Frank theory, the size of the defect core relative to the particle did not enter the calculations. As such the influence of confinement was not considered, with the results corresponding to the unconfined case. The Landau-de Gennes theory reveals that the twist associated with such a companion defect (that exists typically as a loop) extends over a length-scale comparable to the particle radius, and the amount of twist and the twist transition itself depend on the size of the defect core relative to the particle radius [24].

In this paper the analysis of Rüdinger and Stark [20] is extended to the case of an idealized hyperbolic hedgehog. We aim to show how the elastic moduli and the degree of confinement affect the stability of a hyperbolic hedgehog in deciding whether it is twisted or not.

The ground state of the liquid crystal is assumed to be an "ideal" untwisted hyperbolic hedgehog defined by the director field $\hat{n}_{0}=(-x,-y, z) /|(x, y, z)|$ and constrained between two concentric spheres (so as to circumvent the defect core) with 


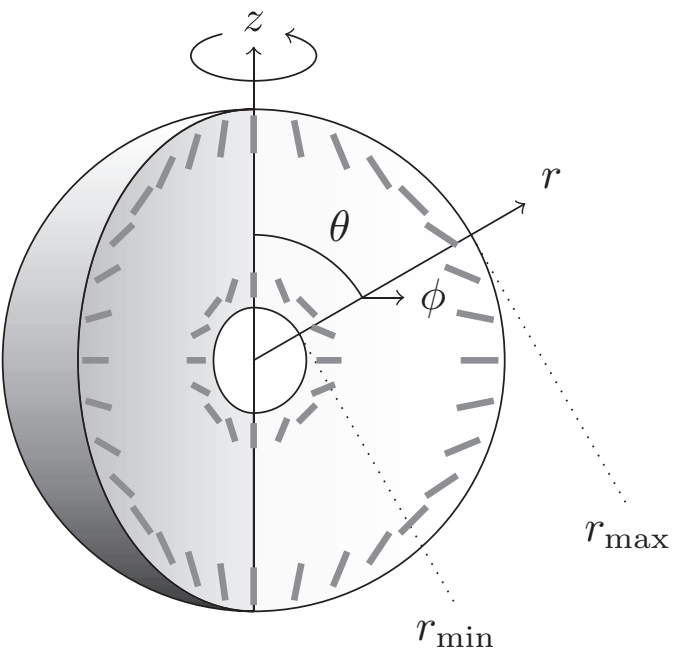

FIG. 1. Geometry of an idealized hyperbolic-hedgehog confined within a thick shell extending from $r_{\min }$ to $r_{\max }$, where the gray bars represent the fixed surface director orientation. After the twist transition, the director rotates in the direction of $\hat{e}_{\phi}$.

an inner radius of $r_{\min }$ and an outer radius of $r_{\max }$, as sketched in Fig. 1. It should be noted that although such an isolated entity is contrived, it is convenient for studying the effect of confinement. In reality hyperbolic defects are seen in more complex configurations, most commonly in liquid crystal colloids [21]. by

The Frank free energy of a nematic liquid crystal is given

$$
\begin{aligned}
\mathcal{F}= & \frac{1}{2} \int d V\left\{k_{11}(\nabla \cdot \hat{n})^{2}+k_{22}(\hat{n} \cdot \nabla \times \hat{n})^{2}\right. \\
& \left.+k_{33}[\hat{n} \times(\nabla \times \hat{n})]^{2}\right\},
\end{aligned}
$$

where surface terms have been neglected, since the director is assumed fixed at $r_{\min }$ and $r_{\max }$. Substituting the director field of the ideal hedgehog yields the free energy $(8 / 15) \pi\left(3 k_{11}+\right.$ $\left.2 k_{33}\right)\left(r_{\max }-r_{\min }\right)$.

Strictly speaking, in a shell of liquid crystal where the orientation is fixed at the interior and exterior boundaries and set to follow the ideal hyperbolic-hedgehog, $\hat{n}_{0}$, the ideal hyperbolic hedgehog is only obtained in the interior when $k_{11}=k_{33}$. When $k_{11}$ and $k_{33}$ differ, the director field found by minimizing the free energy exhibits polar (bendsplay) deformation relative to the ideal case, most prominently in the vicinity of the inner boundary. The magnitude of the relative deformation varies continuously as a function of $k_{11} / k_{33}$; in other words, a transition is not observed and the deformation is relatively small. For instance, with $k_{11} / k_{33}=$ $1 / 2$ the tilt deviates by $\pm 4^{\circ}$ compared to the ideal hedgehog, causing a $3 \%$ relative drop in the free energy (calculated by the method outlined in Sec. III).

Section II proceeds under the assumption that the ideal hyperbolic hedgehog is a good approximation of the ground state, neglecting any bend-splay distortion from the ideal that arises due to the elastic anisotropy. This simplifies the analysis significantly since $\hat{n}_{0}$ is independent of the radius, $r$. In Sec. III the twist transition is reevaluated allowing for such bend-splay distortions by nonlinear analysis. Finally, Sec. IV investigates whether or not the earlier analyses can be applied to a wider class of geometries by considering the twist transition of a hyperbolic hedgehog defect associated with a spherical particle.

\section{LINEAR STABILITY ANALYSIS}

Following the approach of Rüdinger and Stark [20], the local director $\hat{n}$ is represented in spherical coordinates, allowing for small deviations in the azimuthal, $\phi$, direction from the ground state,

$$
\hat{n}(r, \theta)=\left(1-\frac{1}{2} b^{2} f^{2}\right)\left[\cos (2 \theta) \hat{e}_{r}-\sin (2 \theta) \hat{e}_{\theta}\right]+b f \hat{e}_{\phi},
$$

where $f(r, \theta)$ is a general function and $b$ is the magnitude of the azimuthal (twist) deviation from the ideal hyperbolic hedgehog. The $\cos (2 \theta)$ and $\sin (2 \theta)$ terms lead to more complex expressions than seen in the radial case [20]. Simplicity could be restored through the use of hyperbolic coordinates, but the natural boundary surface would also be hyperbolic in shape and inconvenient for the study of confined systems.

After substituting $\hat{n}(r, \theta)$ into the free energy and keeping terms up to second order in $b$ (linear terms are absent), the free-energy difference relative to the untwisted case can be written as

$$
\begin{aligned}
\Delta \mathcal{F}= & \pi b^{2} \int_{r_{\min }}^{r_{\max }} d r \int_{0}^{\pi} \sin \theta d \theta\left(\left[k_{22} \cos ^{2}(2 \theta)+k_{33} \sin ^{2}(2 \theta)\right] f_{\theta}^{2}+\left(k_{22}-k_{33}\right) \sin (4 \theta) r f_{r} f_{\theta}\right. \\
& +\left[k_{22} \sin ^{2}(2 \theta)+k_{33} \cos ^{2}(2 \theta)\right] r^{2} f_{r}^{2}-\left[4 k_{11} \cos ^{2} \theta+k_{22}\left(4 \sin ^{2} \theta-\csc ^{2} \theta\right)-2 k_{33}\right] \sin (2 \theta) f f_{\theta} \\
& +\left\{4 k_{11} \cos ^{2} \theta+4 k_{22}[2 \sec (2 \theta)-1] \cos ^{2} \theta-2 k_{33}\right\} \cos (2 \theta) r f f_{r} \\
& \left.-\left\{4 k_{11} \cos ^{4} \theta+k_{22}\left(4 \sin ^{4} \theta-\csc ^{2} \theta-3\right)+k_{33}\left[2 \sin ^{2}(2 \theta)+2 \cos (2 \theta)+1\right]\right\} f^{2}\right),
\end{aligned}
$$

where $f_{r}=\partial f / \partial r$ and $f_{\theta}=\partial f / \partial \theta$. Here, due to the second term in $f_{r} f_{\theta}, f(r, \theta)$ cannot be written as the product of two independent functions of $r$ and $\theta$. In contrast, $f(r, \theta)$ is separable in the radial hedgehog case. To alleviate this problem, the canonical form of the second-order elliptic partial differential equation given by taking the first variation in $\Delta \mathcal{F}$ is sought. This suggests the following substitution for $r$,

$$
\alpha=\frac{1}{4} \ln \left[1+\left(k_{22} / k_{33}-1\right) \cos ^{2}(2 \theta)\right]+\ln (r) .
$$

It is now natural to set boundary conditions at fixed values of $\alpha$ and $\theta$. A contour of fixed $\alpha$ forms a rounded diamond in $r-\theta$ space, with an eccentricity that depends on $k_{22} / k_{33}$. 
After substituting $r$ for $\alpha$ and, since $b$ is arbitrary, the following inequality is obtained which, if satisfied, informs us that the hyperbolic hedgehog will be twisted,

$$
\begin{aligned}
& \int_{\alpha_{\min }}^{\alpha_{\max }} e^{\alpha} d \alpha \int_{-1}^{1}\left[1+\left(\frac{k_{22}}{k_{33}}-1\right) \cos ^{2}(2 \theta)\right]^{-1 / 4} d \cos \theta\left\{\left[k_{22} \cos ^{2}(2 \theta)+k_{33} \sin ^{2}(2 \theta)\right] \bar{f}_{\theta}^{2}\right. \\
& \quad+\left[k_{22} \cos ^{2}(2 \theta)+k_{33} \sin ^{2}(2 \theta)\right]^{-1} k_{22} k_{33} \bar{f}_{\alpha}^{2}+\left[\left(k_{11}-k_{22}\right)\left(12 \cos ^{4} \theta-10 \cos ^{2} \theta\right)\right. \\
& \left.\left.\quad+\left(k_{33}-k_{22}\right)\left(8 \sin ^{4} \theta-6\right)+k_{22}\left(\csc ^{2} \theta-2\right)\right] \bar{f}^{2}\right\}<0
\end{aligned}
$$

This has solutions $\bar{f}(\alpha, \cos \theta)=f(r, \theta)$, subject to Eq. (4). In the above expression further simplifications have been made, namely the first-order terms in $\bar{f} \bar{f}_{\alpha}$ and $\bar{f} \bar{f}_{\theta}$ have been eliminated. The terms in $\bar{f} \bar{f}_{\alpha}$ and $\bar{f} \bar{f}_{\theta}$ are rewritten in a form to which the divergence theorem can be applied. This procedure gives rise to additional terms in $\bar{f}^{2}$. After applying the divergence theorem, boundary terms result that also depend on $\bar{f}^{2}$. Following Ref. [20] we consider Dirichlet boundary conditions for $\bar{f}(\alpha, \cos \theta)$ at the boundaries, specifically $\bar{f}\left(\alpha_{\min }, \cos \theta\right)=\bar{f}\left(\alpha_{\max }, \cos \theta\right)=\bar{f}(\alpha,-1)=\bar{f}(\alpha, 1)=0$. Due to these fixed boundary conditions, the boundary terms in $\bar{f}^{2}$ can be dropped since they do not contribute to the free energy.

The left-hand side remains proportional to the free energy relative to the untwisted state in the transformed coordinate system. At this stage the coordinate transformation $x=\cos \theta$ is applied. After applying Green's theorem and taking into account the boundary conditions, the inequality becomes

$$
\frac{\int_{\alpha_{\min }}^{\alpha_{\max }} d \alpha \int_{-1}^{1} d x r \bar{f}\left(\mathcal{D}_{\alpha}+\mathcal{D}_{x}\right) \bar{f}}{\int_{\alpha_{\min }}^{\alpha_{\max }} d \alpha \int_{-1}^{1} d x r \bar{f}^{2}}<2 k_{22} .
$$

The inequality Eq. (6) is best fulfilled when its left-hand side assumes a minimum. This minimum, according to the Ritz principle in quantum mechanics, is given by the lowest eigenvalue of the operator $\mathcal{D}_{\alpha}+\mathcal{D}_{x}$ subject to the boundary conditions given earlier [20]. The eigenvalue equation $\mathcal{D}_{\alpha} \bar{f}+$ $\mathcal{D}_{x} \bar{f}=\lambda \bar{f}$ is separable into a radial part and an angular part, with eigenfunctions defined as $\bar{f}(\alpha, x)=g(\alpha) h(x)$.

For the radial dependence, we seek the lowest eigenvalue of the operator $\mathcal{D}_{\alpha}=-\partial^{2} / \partial \alpha^{2}-\partial / \partial \alpha$, which is

$$
\lambda_{\alpha}=\frac{1}{4}+\frac{\pi^{2}}{\left(\alpha_{\max }-\alpha_{\min }\right)^{2}},
$$

and corresponds to the eigenfunction

$$
g(\alpha)=\exp \left(-\frac{\alpha}{2}\right) \sin \left(\pi \frac{\alpha-\alpha_{\min }}{\alpha_{\max }-\alpha_{\min }}\right) .
$$

When $k_{22}=k_{33}$, this solution is identical to that found for the radial hedgehog [20]. A sketch of this function may be found in Ref. [20, Fig. 4].

The function $g(\alpha)$ contributes to the free energy in the following fashion:

$$
\begin{gathered}
\int_{\alpha_{\min }}^{\alpha_{\max }} e^{\alpha} g(\alpha)^{2} d \alpha=\frac{1}{2}\left(\alpha_{\max }-\alpha_{\min }\right), \\
\int_{\alpha_{\min }}^{\alpha_{\max }} e^{\alpha} g_{\alpha}(\alpha)^{2} d \alpha=\frac{1}{2}\left(\alpha_{\max }-\alpha_{\min }\right) \lambda_{\alpha} .
\end{gathered}
$$

The angular dependence can be derived from the lowest eigenvalue of $\mathcal{D}_{x}$ defined as

$$
\begin{aligned}
\mathcal{D}_{x}= & -\left[k_{22}-4\left(k_{22}-k_{33}\right)\left(1-x^{2}\right) x^{2}\right]\left(1-x^{2}\right) \partial^{2} / \partial x^{2} \\
& +\left[k_{22}+\left(k_{22}-k_{33}\right)\left(1-x^{2}\right)\left(3-10 x^{2}\right)\right] 2 x \partial / \partial x \\
& +\left[k_{22}-4\left(k_{22}-k_{33}\right)\left(1-x^{2}\right) x^{2}\right]^{-1} k_{22} k_{33} \lambda_{\alpha} \\
& +\left(k_{11}-k_{22}\right)\left(12 x^{4}-10 x^{2}\right) \\
& +\left(k_{33}-k_{22}\right)\left(8 x^{4}-16 x^{2}+2\right) \\
& +k_{22}\left(1-x^{2}\right)^{-1} .
\end{aligned}
$$

It is clear that all three elastic constants, as well as the degree of confinement (provided $k_{22} \neq k_{33}$ ) acting via $\lambda_{\alpha}$, affect $h(x)$. For the radial hedgehog, the situation is much simpler; the minimum eigenvalue solution is independent of these parameters. In the one elastic constant case the differential operator is identical to that found in the radial case, namely the associated Legendre differential equation, which has solutions $P_{\ell}^{m}(x)$, where $P_{\ell}^{m}(x)$ are the associated Legendre polynomials. For Eq. (10), $m=1$. The minimum eigenvalue occurs when $\ell=1$ and its corresponding eigenfunction is $h(x)=P_{1}^{1}(x)$.

In order to find how perturbations to the elastic constants from the one elastic constant case affect $h(x)$ to first order, the differential operator is expanded as

$$
\mathcal{D}_{x}=\mathcal{D}_{x}^{(0)}+\epsilon \mathcal{D}_{x}^{(1)},
$$

where $\epsilon$ is presumed to be small, $\mathcal{D}_{x}^{(0)}$ is equal to $\mathcal{D}_{x}$ in the case of equal elastic constants, and $\mathcal{D}_{x}^{(1)}$ is the change in $\mathcal{D}_{x}$ caused by a perturbation equal to $\epsilon$ in one of the elastic constants. Note that when $k_{22}=k_{33}$, the $\lambda_{\alpha}$ term contributes a constant amount, which serves to shift the eigenvalue but has no effect on the eigenfunction itself.

Change in a given eigenfunction due to the perturbation can be written

$$
h_{\ell}(x)=h_{\ell}^{(0)}(x)+\epsilon h_{\ell}^{(1)}(x),
$$

where $h_{\ell}^{(0)}(x)=P_{\ell}^{1}(x)$ and

$$
h_{\ell}^{(1)}(x)=\sum_{n(\neq \ell)} \frac{h_{n}^{(0)}(x)}{\lambda_{\ell}^{(0)}-\lambda_{n}^{(0)}} \frac{\int_{-1}^{1} h_{n}^{(0)}(y) \mathcal{D}_{x}^{(1)} h_{\ell}^{(0)}(y) d y}{\int_{-1}^{1}\left[h_{n}^{(0)}(y)\right]^{2} d y} .
$$

For the (scaled) associated Legendre Eq. (10) the unperturbed eigenvalues are given by $\lambda_{n}^{(0)}=k \lambda_{\alpha}+k n(n+1)$, where $k$ is the elastic constant in the unperturbed case, which we approximate by $k=\left(k_{11}+k_{22}+k_{33}\right) / 3$.

Difficulty in integrating $\left[k_{22}-4\left(k_{22}-k_{33}\right)\left(1-x^{2}\right) x^{2}\right]^{-1}$ of the $\lambda_{\alpha}$ term is alleviated by replacing it with its Taylor expansion. In the case of a perturbation in $k_{22}$, the Taylor expansion is taken with respect to $k_{22}$ about $k_{22}=k$. Keeping 
up to first-order terms (in $\epsilon$ ) gives $4\left(1-x^{2}\right) x^{2} \lambda_{\alpha}$ as the term's contribution to $\mathcal{D}_{x}^{(1)}$. Similarly for a perturbation in $k_{33}$, the expansion with respect to $k_{33}$ about $k_{33}=k$ yields a contribution of $\left[1-4\left(1-x^{2}\right) x^{2}\right] \lambda_{\alpha}$.

Of principal interest is the change due to the perturbation in the eigenfunction with the lowest eigenvalue, $\ell=1$, for which nonzero terms in the above series arise only when $n=3$ or $n=5$, such that

$$
h(x)=P_{1}^{1}(x)+a_{3} P_{3}^{1}(x)+a_{5} P_{5}^{1}(x) .
$$

Summing contributions to the coefficients, $a_{k}$, from perturbations in $k_{11}, k_{22}$, and $k_{33}$ considered separately gives

$$
\begin{aligned}
& a_{3}=\frac{2}{225 k}\left[3 k_{11}-\left(24+2 \lambda_{\alpha}\right) k_{22}+\left(21+2 \lambda_{\alpha}\right) k_{33}\right], \\
& a_{5}=\frac{8}{2205 k}\left[-3 k_{11}+\lambda_{\alpha} k_{22}+\left(3-\lambda_{\alpha}\right) k_{33}\right],
\end{aligned}
$$

revealing that $a_{3}$ plays a dominant role on $h(x)$ and that $a_{3}$ itself depends largely on the values of $k_{22}$ and $k_{33}$.

Although ideally the full analytic solution to the differential operator should be sought, its complexity makes this a challenge. Since our ultimate aim is to derive an equation for the twist inequality (although we resort to approximations due to the complexities of the expressions involved), the perturbation method desirably gives a simple linear relationship between the elastic constants and the coefficients $a_{k}$. Later the validity of the approximation is confirmed. An alternative approach is to substitute the series $h(x) \approx \sum_{n=1}^{N} a_{2 n-1} P_{2 n-1}^{1}(x)$ into the operator and solve for the coefficients directly, but this gives complicated terms even for the modest choice $N=3$.

The approximation for $h(x)$ given by the perturbation method works particularly well for $k_{11} / k$ and $k_{33} / k$ but less so for $k_{22} / k$. Here, benefit could be seen by considering higher-order terms in the perturbation. Figure 2 compares this approximation to the numerical solution (calculated by a pseudospectral method) with elastic constants corresponding to the liquid crystalline material $5 \mathrm{CB}$. Although the elastic

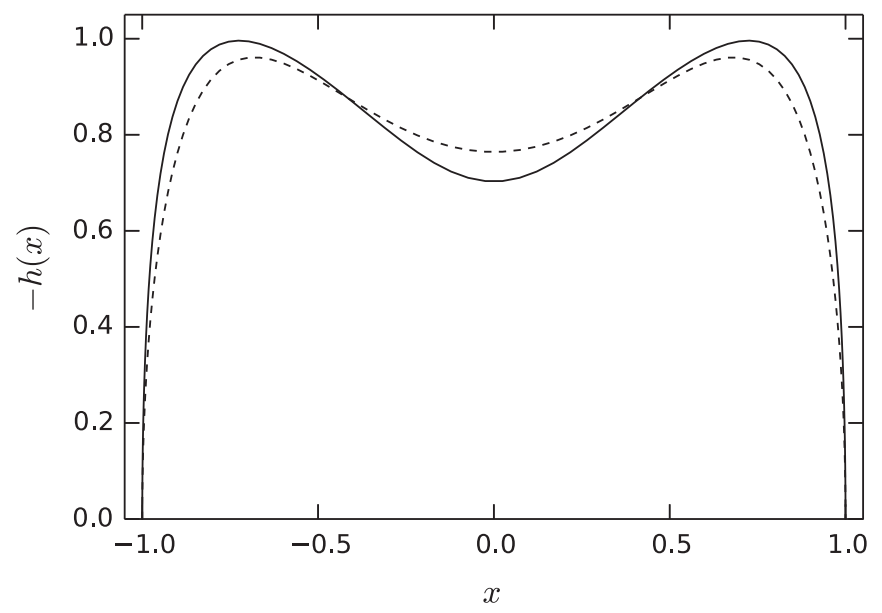

FIG. 2. Angular dependence of $\bar{f}(\alpha, x), h(x)$, (minimum eigenvalue solution) when $\alpha_{\max }-\alpha_{\min } \rightarrow \infty$ for $5 \mathrm{CB}$, with $k_{11} / k_{33}=$ 0.79 and $k_{22} / k_{33}=0.43$. Solid line is the full numerical solution [normalized to conform to the form of Eq. (14)] and the dotted line is the approximation given by first-order perturbation analysis. constant ratios deviate strongly from unity for this material, the approximation remains reasonable. Furthermore, the validity of the linear analysis is limited to the vicinity of the twist transition, whereabouts the deviation in the elastic moduli is expected to be less extreme.

With $\bar{f}(\alpha, x)$ fully approximated, it is now possible to evaluate the free energy and determine whether a set of elastic constants satisfy the twist inequality Eq. (5). At this stage, two further approximations are made for $h(x)$. By assuming $k_{11}=$ $\left(k_{22}+k_{33}\right) / 2$ and $\lambda_{\alpha}=1 / 4$ in evaluating the coefficients $a_{k}$, the variational inequality is simplified significantly; it becomes linear in $k_{11}$ and $\lambda_{\alpha}$.

Subsequently, $h(x)$ is multiplied by $k / k_{33}$ before it is substituted into the inequality Eq. (5). In evaluating the integrals that appear in the inequality, the $\left[1+\left(k_{22} / k_{33}-\right.\right.$ 1) $\left.\cos ^{2}(2 \theta)\right]^{-1 / 4}$ term due to the coordinate transformation is difficult to tackle directly. Instead it is replaced by its Taylor expansion in $k_{22} / k_{33}$ about $k_{22} / k_{33}=1$, which is $1-(1 / 4)\left(k_{22} / k_{33}-1\right) \cos ^{2}(2 \theta)$ to first order. Substitution is similarly made for the $\left[1+\left(k_{22} / k_{33}-1\right) \cos ^{2}(2 \theta)\right]^{-1}$ term. In both cases, it proves sufficient to keep terms up to first order in $k_{22} / k_{33}$.

Finally, the twist inequality is divided by $k_{33}$ and integrated with respect to $\theta$. Solving for $k_{11} / k_{33}$ yields a rational polynomial in $k_{22} / k_{33}$. If $k_{11} / k_{33}$ exceeds this polynomial, the hedgehog will be twisted. Before truncating this polynomial we substitute $k_{22} / k_{33}=\exp (\bar{k})$, motivated by the fact that the numerical solution for the threshold value of $k_{11} / k_{33}$ at which twist occurs depends almost linearly on the logarithm of $k_{22} / k_{33}$. Taking the Taylor expansion of the rational polynomial in terms of $\bar{k}$ about $\bar{k}=0$ and keeping terms up to first order yields

$$
\frac{k_{11}}{k_{33}}>\left(\frac{230276}{333795} \lambda_{\alpha}+\frac{21}{17}\right) \ln \left(\frac{k_{22}}{k_{33}}\right)+\frac{35}{34} \lambda_{\alpha}+1 .
$$

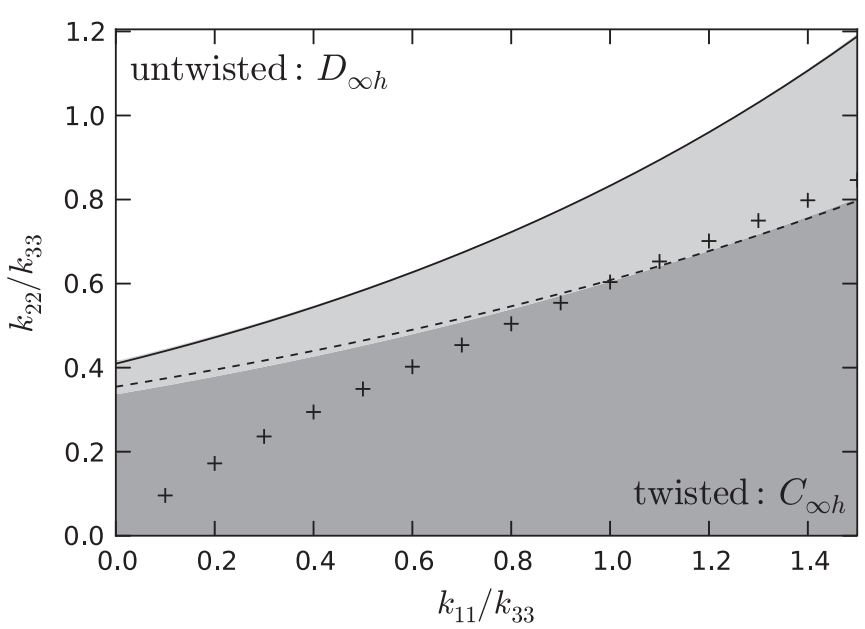

FIG. 3. White region indicates where the untwisted hyperbolic configuration is stable as (i) $\alpha_{\max }-\alpha_{\min } \rightarrow \infty$, calculated numerically. Dark-gray region indicates the stability region of the twisted hyperbolic configuration with (ii) $\alpha_{\max }-\alpha_{\min }=\ln (50)$. Solid and dotted lines correspond to the analytic approximation of Eq. (16) for (i) and (ii). (+) Threshold value of $k_{22} / k_{33}$ below which twist occurs with $r_{\max } / r_{\min }=50$ given by the nonlinear analysis of Sec. III. 
Figure 3 shows the instability domain of the untwisted hyperbolic hedgehog with respect to a twist transition as a function of the elastic moduli, where both $h(x)$ and the twist inequality are evaluated numerically. Within the domain the hedgehog is twisted. When $\alpha_{\max }-\alpha_{\min } \rightarrow \infty$ the domain is at its largest and corresponds to the union of the gray patches. As the degree of confinement increases to $\alpha_{\max }-\alpha_{\min }=\ln (50)$, which corresponds to $r_{\max } / r_{\min } \approx 50$, the domain shrinks to the dark-gray patch. The light-gray patch indicates where the untwisted hedgehog is unstable for $\alpha_{\max }-\alpha_{\min }>\ln (50)$, but stable for $\alpha_{\max }-\alpha_{\min }=\ln (50)$.

Solid and dotted lines correspond to the approximation Eq. (16), which agrees well with the numerical solution. For $\alpha_{\max }-\alpha_{\min } \rightarrow \infty$, the thresholds as plotted are indistinguishable, but a slight departure is apparent for $\alpha_{\max }-\alpha_{\min }=$ $\ln (50)$, which is only reduced marginally by keeping terms of higher order than first in Eq. (16). The root cause of the departure (and slowed convergence; first-order terms should suffice) is the approximation used for $h(x)$ given by the first-order perturbation analysis.

Clearly, $\alpha_{\max }$ and $\alpha_{\min }$ or equivalently $r_{\max }$ and $r_{\min }$ play a critical role on the size of the instability domain. The extent of the confining geometry sets the value of $r_{\max }$, whereas $r_{\text {min }}$ relates to the size of the defect core and is not so straightforward to estimate. At the core exists a defect loop, inside which the director is approximately uniform. Twist tends to zero at the loop origin, but peaks in the vicinity of the loop radius. Therefore, we suggest $r_{\min }$ is approximately proportional to the loop radius, which has been established as function of the elastic moduli by Fukuda and Yokoyama [12].

\section{NONLINEAR STABILITY ANALYSIS}

In a shell with fixed boundary conditions corresponding to an ideal hyperbolic hedgehog, there is inevitably some deviation in tilt internally from the ideal when $k_{11}$ and $k_{33}$ differ. When they differ appreciably, the tilt deviation can no longer be assumed small, and a simple linear analysis breaks down. In this section, the stability criterion for the twisted hyperbolic hedgehog is reevaluated, allowing for deviations in the tilt from the ideal hedgehog, by nonlinear analysis. The director field may be written as

$$
\hat{n}(r, \theta)=\cos (\chi)\left[\cos (2 \psi) \hat{e}_{r}-\sin (2 \psi) \hat{e}_{\theta}\right]+\sin (\chi) \hat{e}_{\phi}
$$

where the tilt and twist are represented by the general functions $\psi$ and $\chi$, respectively.

Once again a spherical coordinate system is assumed. When $\hat{n}(r, \theta)$ is substituted into the free-energy Eq. (1), mixed derivative terms once again arise for both $\chi$ and $\psi$, which cannot be eliminated by the same coordinate transform. Instead we proceed using $\alpha=\ln (r)$, which enables the $r$ dependence of $\chi$ to be well resolved when discretization is performed.

At the boundaries the twist is assumed to be zero, $\chi\left(\alpha_{\min }, \theta\right)=\chi\left(\alpha_{\max }, \theta\right)=\chi(\alpha, 0)=\chi(\alpha, \pi)=0$. The tilt is assumed to follow the ideal hedgehog at the boundaries so that $\psi(\alpha, \theta)=\theta$.
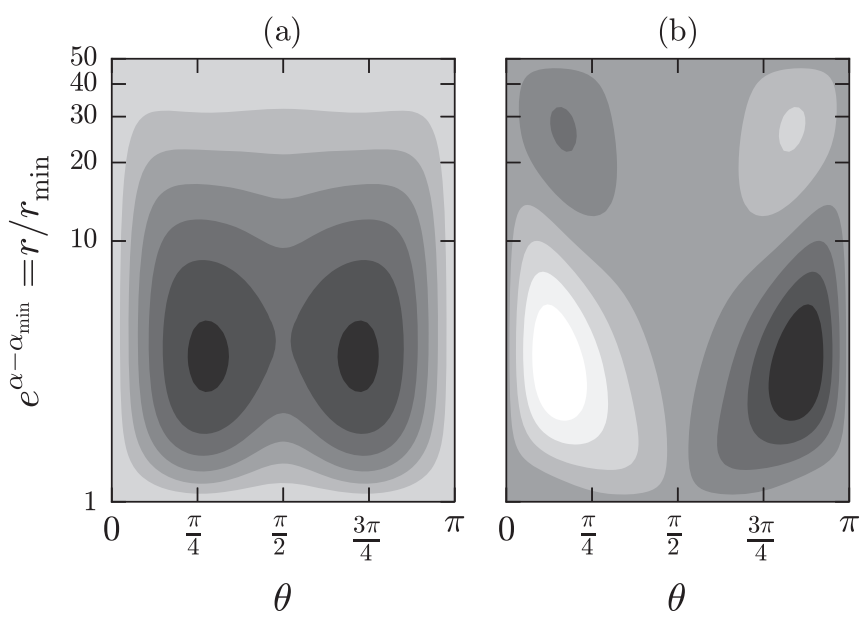

FIG. 4. (a) Twist, $\chi(\alpha, \theta)$, and (b) tilt relative to the ideal hedgehog, $\psi(\alpha, \theta)-\theta$, of a hyperbolic hedgehog given by the nonlinear analysis assuming the elastic constants of 5CB and $\alpha_{\max }-\alpha_{\min }=\ln (50)$. Minimum is shaded white and the maximum black. Peak twist is $28.3^{\circ}$ and deviation in tilt is at most $\pm 2.4^{\circ}$.

In the transformed coordinate system, the spatial derivative terms in $\hat{n}$ that appear in the free energy are given by

$$
\begin{aligned}
\frac{e^{\alpha} \nabla \cdot \hat{n}}{\cos \chi}= & \left(\chi_{\theta} \tan \chi-2 \psi_{\alpha}-\cot \theta\right) \sin (2 \psi) \\
& +\left(2-\chi_{\alpha} \tan \chi-2 \psi_{\theta}\right) \cos (2 \psi), \\
\frac{e^{\alpha} \nabla \times \hat{n}}{\cos \chi}= & {\left[\chi_{\theta}+\tan \chi \cot \theta\right] \hat{e}_{\alpha}-\left[\chi_{\alpha}+\tan \chi\right] \hat{e}_{\theta} } \\
& +\left[\left(\chi_{\alpha} \tan \chi+2 \psi_{\theta}-1\right) \sin (2 \psi)\right. \\
& \left.+\left(\chi_{\theta} \tan \chi-2 \psi_{\alpha}\right) \cos (2 \psi)\right] \hat{e}_{\phi} .
\end{aligned}
$$

Taking the first variation in the free energy leads to the Euler-Langrange equations for $\chi$ and $\psi$, which are solved using a pseudospectral method on a $31 \times 31$ point grid defined by the tensor product of Chebyshev-Gauss-Lobatto points along $\alpha$ and $\theta$. Simultaneously, the effectively twodimensional problem for $\psi$ and $\chi$ is solved in linearized form using Newton's method.

At the twist threshold, $\chi$ is small and negligibly affects $\psi$. Consequently, one may solve for $\chi$ and $\psi$ independently. However, this approach is not taken herein. Instead, the full coupled system is solved.

In Fig. 4 the resulting twist and tilt of a hyperbolic hedgehog are shown for 5CB. The twist, $\chi(\alpha, \theta)$, is similar to that given by the earlier linear analysis and the tilt $\psi(\alpha, \theta)$ departs from $\theta$ by $\pm 2.4^{\circ}$ [in the case of the ideal hedgehog $\psi(\alpha, \theta)=\theta$ ].

The twist transition is second-order [20,23] and can be characterized by an order parameter, $\chi_{\max }$, defined as the maximum value taken by $\chi$. Associated with the transition is a breaking of symmetry from $D_{\infty h}$ to $C_{\infty h}$. For a given value of $k_{11} / k_{33}$ and in the vicinity of the transition $\chi_{\max } \propto$ $\sqrt{\left[k_{22} / k_{33}\right]_{\text {th }}-k_{22} / k_{33}}$, where $\left[k_{22} / k_{33}\right]_{\text {th }}$ is the threshold value of $k_{22} / k_{33}$ beneath which twist occurs. By means of this formula or using the bisection method $\left[k_{22} / k_{33}\right]_{\text {th }}$ can be determined efficiently.

In Fig. 3 the threshold values given by the nonlinear analysis are plotted as pluses. At $k_{11} / k_{33}=1$ the threshold 
(a)

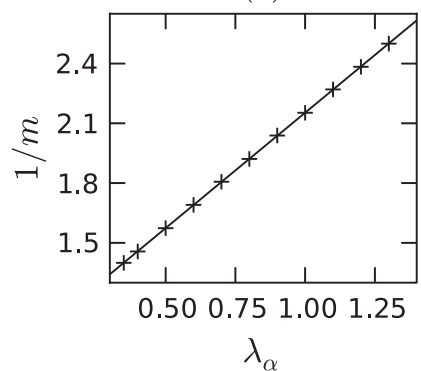

(b)

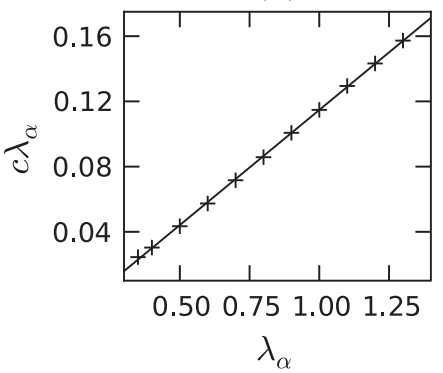

FIG. 5. Twist transition threshold given by the nonlinear analysis can be fitted by $k_{22} / k_{33}<m k_{11} / k_{33}+c$ for a given value of $\lambda_{\alpha}$, where $m$ and $c$ are evaluated at $k_{11} / k_{33}=1$. (+) Numerical values of (a) $1 / m$ and (b) $c \lambda_{\alpha}$ as functions of $\lambda_{\alpha}$, well approximated by the linear fits (-) given by (a) $1 / m=1.159 \lambda_{\alpha}+0.994$, (b) $c \lambda_{\alpha}=0.141 \lambda_{\alpha}-0.027$.

is almost identical to that given by the linear analysis of Sec. II. The marginal difference, $0.2 \%$ for $\alpha_{\max }-\alpha_{\min }=$ $\ln (50)$ (which is more pronounced in highly confined cases), is due to the geometry difference arising from the differing definitions of $\alpha$. The threshold can be well fitted by a straight line $k_{22} / k_{33}<m k_{11} / k_{33}+c$. This linear dependence can be directly attributed to the tilt deformation; if the tilt angle is fixed to coincide with the ideal hedgehog, $\psi=\theta$, the threshold is identical to the earlier linear analysis. The same linear relationship was found by Stark [23] for the twist transition of a hyperbolic companion defect about a spherical particle.

To assess how the degree of confinement affects the transition, $\lambda_{\alpha}$ proves to be a useful measure, defined identically to Eq. (7). Figure 5 reveals that $1 / m$ and $c \lambda_{\alpha}$ (evaluated at $\left.k_{11} / k_{33}=1\right)$ as functions of $\lambda_{\alpha}$ can be well fitted by straight lines. by

From the linear fits, the twist transition can be approximated

$$
\frac{k_{22}}{k_{33}}<\frac{1}{1.159 \lambda_{\alpha}+0.994} \frac{k_{11}}{k_{33}}-0.027 \frac{1}{\lambda_{\alpha}}+0.141 .
$$

For comparison, the twist transition reported by Rüdinger and Stark [20] for a radial hedgehog within a droplet is $k_{22} / k_{33}<k_{11} / k_{33}-\lambda_{\alpha} / 2$. Typically, calamitic liquid crystals satisfy $k_{33} \geqslant k_{11}>k_{22}$. When $k_{11} / k_{33}=1$, the hyperbolic hedgehog twists more readily than the radial (that is to say, the twist transition occurs at a larger value of $\left.k_{22} / k_{33}\right)$ provided $\alpha_{\max }-\alpha_{\min }<\ln (129)$. Whereas with $k_{11} / k_{33}<0.71$ [estimated by Eq. (19)], the hyperbolic hedgehog twists more readily for all $\lambda_{\alpha}$. In part this increased tendency to twist can be attributed to the fact that the free energy of the untwisted configuration depends on $k_{11}$ alone in the radial case, but on both $k_{11}$ and $k_{33}$ in the hyperbolic. Hyperbolic hedgehogs alone suffer the energetically costly bend and more readily twist to alleviate it.

\section{TWIST TRANSITION OF A COMPANION DEFECT ABOUT A SPHERICAL PARTICLE}

This section, in contrast to those previous, considers a geometry that contains a hyperbolic hedgehog which is easy to realize in practice; a liquid-crystal colloid. This is the most common setting in which hyperbolic hedgehogs are observed. Here, a spherical particle in the dipole configuration is considered in isolation. The twist transition of the hyperbolic hedgehog that accompanies the particle is found and compared to the previous analyses.

In order to study the influence of the defect size on the twist transition using the Oseen-Frank theory, the defect is enclosed within a sphere of cutoff radius $r_{\min }$. At the sphere's surface, fixed hyperbolic alignment is assumed. Nearby a larger sphere of radius $r_{\max }$ represents the particle, with fixed homeotropic alignment at its surface. Finally, at infinity planar alignment is assumed along $\hat{e}_{z}$.

Bispherical coordinates are a natural setting in which to study such a pair of spheres within an unbounded domain [25-27]. Bispherical coordinates $(\zeta, \mu, \phi)$ can be related to cylindrical coordinates $(\rho, z, \phi)$ by

$$
\rho=\frac{a \sin \mu}{\cosh \zeta-\cos \mu}, \quad z=\frac{a \sinh \zeta}{\cosh \zeta-\cos \mu},
$$

for $\mu \in[0, \pi]$. Limits for $\zeta \in\left[\zeta_{1}, \zeta_{2}\right]$ are set according to the particle and cut-off radii as

$$
\zeta_{1}=-\sinh ^{-1}\left(\frac{a}{r_{\max }}\right), \quad \zeta_{2}=\sinh ^{-1}\left(\frac{a}{r_{\min }}\right)
$$

with $a$ given by

$$
a=\frac{1}{2 r_{d}} \sqrt{r_{d}^{4}-2 r_{d}^{2}\left(r_{\max }^{2}+r_{\min }^{2}\right)+\left(r_{\max }^{2}-r_{\min }^{2}\right)^{2}} .
$$

The distance between the particle and defect cores, $r_{d}$, is assumed fixed as $r_{d} / r_{\max }=1.243$ [28]. Although, strictly speaking, $r_{d}$ is a function of the elastic moduli $[23,28]$, it is found to affect the twist transition negligibly $\left(\left[k_{22} / k_{33}\right]_{\text {th }}\right.$ increases by 0.012 for $r_{d} / r_{\max }=1.30$ with $k_{11} / k_{33}=1$ and $r_{\max } / r_{\min }=50$ ).

Cylindrical basis vectors for the director field are used, such that

$$
\hat{n}(\zeta, \mu)=\cos \chi\left(\sin \psi \hat{e}_{\rho}+\cos \psi \hat{e}_{z}\right)+\sin \chi \hat{e}_{\phi},
$$

where the tilt and twist are represented by the general functions $\psi(\zeta, \mu)$ and $\chi(\zeta, \mu)$, respectively. This choice, over bispherical basis vectors, is made because $\hat{n} \cdot \hat{e}_{z}$ remains continuous asymptotically in real space (whereas $\hat{n} \cdot \hat{e}_{\zeta}$ is discontinuous

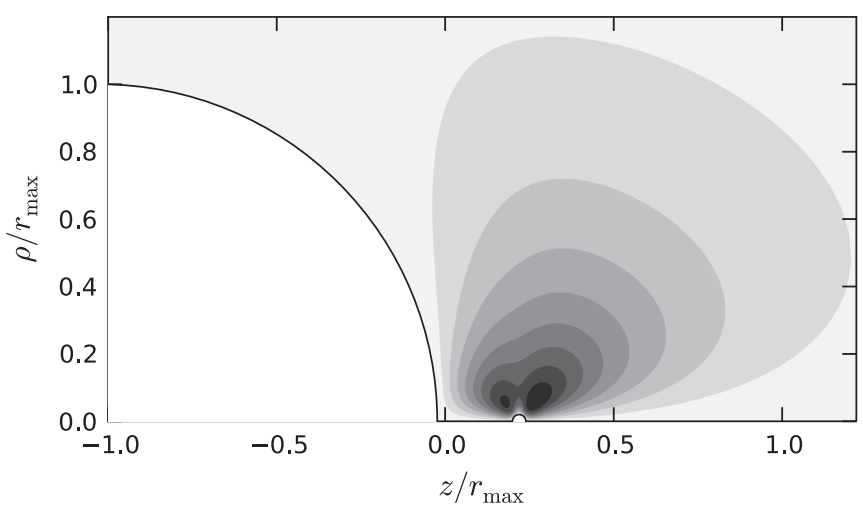

FIG. 6. Twist, $\chi(\zeta, \mu)$, about the dipole state of a spherical particle assuming the elastic constants of 5CB and $r_{\max } / r_{\min }=50$. Peak twist is $30.7^{\circ}$. 
(a)

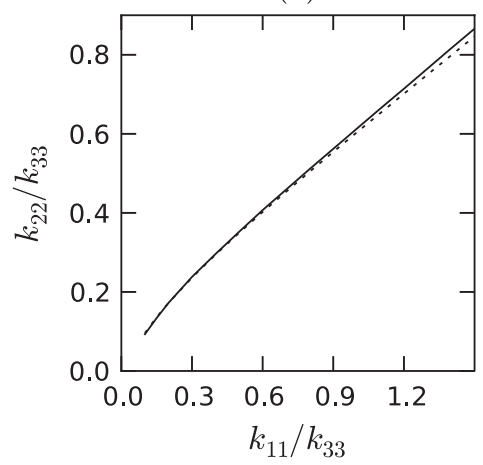

(b)

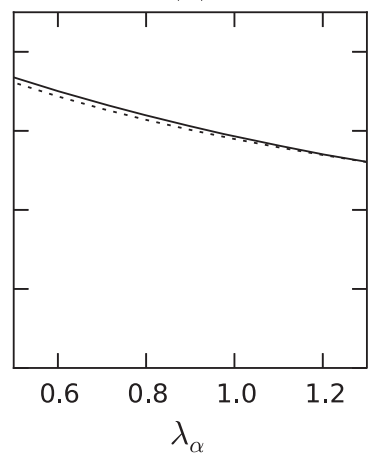

FIG. 7. Threshold value of $k_{22} / k_{33}$ beneath which twist occurs for (-) a companion defect about a particle and (- ) in a hyperbolic shell, using the nonlinear analysis of Sec. III. In (a) $k_{11} / k_{33}$ is varied with $r_{\max } / r_{\text {min }}=50$, whereas in (b) $r_{\max } / r_{\text {min }}$ is varied with $k_{11} / k_{33}=1$.

about infinity). At infinity in real space, where $\zeta=\mu=0$, $\hat{n}(0,0)=\hat{e}_{z}$ is enforced.

The expression for the director is substituted into the free-energy Eq. (1), where the standard expressions for the divergence and curl of $\hat{n}$ in cylindrical coordinates are used with $\partial \hat{n} / \partial \phi=0$, namely $\nabla \cdot \hat{n}=(1 / \rho) \partial\left(\rho \hat{n} \cdot \hat{e}_{\rho}\right) / \partial \rho+\partial(\hat{n}$. $\left.\hat{e}_{z}\right) / \partial z$ and $\nabla \times \hat{n}=\left[-\partial\left(\hat{n} \cdot \hat{e}_{\phi}\right) / \partial z\right] \hat{e}_{\rho}+\left[\partial\left(\hat{n} \cdot \hat{e}_{\rho}\right) / \partial z-\right.$ $\left.\partial\left(\hat{n} \cdot \hat{e}_{z}\right) / \partial \rho\right] \hat{e}_{\phi}+\left[(1 / \rho) \partial\left(\rho \hat{n} \cdot \hat{e}_{\phi}\right) / \partial \rho\right] \hat{e}_{z}$. Spatial derivatives are transformed by the chain rule, which yields

$$
\left[\begin{array}{c}
\frac{\partial}{\partial \rho} \\
\frac{\partial}{\partial z}
\end{array}\right]=\frac{1}{a}\left[\begin{array}{cc}
-\sinh \zeta \sin \mu & \cosh \zeta \cos \mu-1 \\
1-\cosh \zeta \cos \mu & -\sinh \zeta \sin \mu
\end{array}\right]\left[\begin{array}{c}
\frac{\partial}{\partial \zeta} \\
\frac{\partial}{\partial \mu}
\end{array}\right]
$$

and the differential volume in bispherical coordinates is given by

$$
d V=\frac{a^{3} \sin \mu}{(\cosh \zeta-\cos \mu)^{3}} d \zeta d \mu d \phi
$$

Euler-Langrange equations for $\psi(\zeta, \mu)$ and $\chi(\zeta, \mu)$ are determined with the help of Sage [29] and are lengthy. Derivatives are evaluated numerically using $5 \times 5$ point finite difference stencils. A $41 \times 61$ grid in $\zeta$ and $\mu$ is chosen that is initially uniform, but shifted to include $\zeta=0$, since $\zeta=\mu=0$ must be present. The resulting nonlinear system of equations is solved using Newton's method, starting from the ansatz for $\psi(\zeta, \mu)$ given by Lubensky et al. [30].

Figure 6 reveals that the twist, $\chi(\zeta, \mu)$, about a hyperbolic defect associated with a particle, much like in the hyperbolic shell of Fig. 4, is double peaked, but in contrast is lopsided.

Comparing $\left[k_{22} / k_{33}\right]_{\text {th }}$ for the particle and shell, as shown in Fig. 7, the agreement is remarkable (within \pm 0.02 ) considering the difference in geometries. The lopsidedness of the twist or the fixed value of $r_{d}$ could account for the discrepancy of \pm 0.02 . Larger values of $r_{\max } / r_{\min }$, or equivalently smaller values of $\lambda_{\alpha}$, than those plotted are better resolved by the nonlinear analysis of Sec. III.

\section{CONCLUSIONS}

The twist transition of an isolated hyperbolic hedgehog has been found by two methods. Linear analysis gives the twist transition Eq. (16) valid in the vicinity of $k_{11} / k_{33}=1$. When $k_{11}$ and $k_{33}$ differ, inevitably some deviation in the bend and splay arises, typically a few degrees relative to the ideal hedgehog. Although the deviation is slight, when taken into account, the twist transition threshold comes to depend linearly on $k_{11} / k_{33}$ and $k_{22} / k_{33}$, as revealed by the nonlinear analysis of Sec. III. Correspondingly, Stark [23] found the same linear relationship for the twist transition of a particle's companion hyperbolic defect.

Remarkably, the prediction of the twist transition given by the nonlinear analysis for a hyperbolic shell appears to be valid for a wider class of geometries containing hyperbolic defects. In particular, we find that the companion hyperbolic defect about a spherical particle, for example, a constituent particle of a liquid-crystal colloid, twists in much the same way. The threshold value of $k_{22} / k_{33}$ is almost identical, where the degree of confinement is redefined as the ratio of the particle radius to the defect core radius. Compared to previous works [6,23], we find that the hyperbolic hedgehog twists less readily, that is to say the twist transition occurs at a lower value of $k_{22} / k_{33}$.

Contrasting twisted hyperbolic and radial hedgehogs, the radial dependence of the twist [with respect to $\alpha$ defined by (4) and $\alpha=\ln (r)$ respectively in each case] is identical and peaks in the vicinity of the defect core. Its angular dependence is more complex in the hyperbolic case, where it comes to depend on the elastic moduli. Increasing the degree of confinement shifts the twist transition for both hedgehogs and can even inhibit twist entirely.

\section{ACKNOWLEDGMENTS}

The authors are grateful to Professor Y. Kimura, Kyushu University, for helpful discussions and to the Japan Society for the Promotion of Science (JSPS) for financial support.
[1] N. D. Mermin, Rev. Mod. Phys. 51, 591 (1979).

[2] H.-R. Trebin, Adv. Phys. 31, 195 (1982).

[3] O. D. Lavrentovich et al., Defects in Liquid Crystals: Computer Simulations, Theory, and Experiments (Kluwer Academic, Dordrecht, 2001).

[4] G. P. Alexander, B. G.-g. Chen, E. A. Matsumoto, and R. D. Kamien, Rev. Mod. Phys. 84, 497 (2012).
[5] M. Kleman and O. D. Lavrentovich, Philos. Mag. 86, 4117 (2006).

[6] O. D. Lavrentovich and E. M. Terentjev, Sov. Phys. JETP 64, 1237 (1986).

[7] A. Sonnet, A. Kilian, and S. Hess, Phys. Rev. E 52, 718 (1995).

[8] S. Mkaddem and E. C. Gartland, Phys. Rev. E 62, 6694 (2000); E. C. Gartland and S. Mkaddem, ibid. 59, 563 (1999). 
[9] H. Mori and H. Nakanishi, J. Phys. Soc. Jpn. 57, 1281 (1988).

[10] S. Kralj and E. G. Virga, J. Phys. A 34, 829 (2001).

[11] J.-i. Fukuda, M. Yoneya, and H. Yokoyama, Phys. Rev. E 65, 041709 (2002).

[12] J.-i. Fukuda and H. Yokoyama, Phys. Rev. E 66, 012703 (2002).

[13] E. M. Terentjev, Phys. Rev. E 51, 1330 (1995).

[14] S. Kralj, E. G. Virga, and S. Žumer, Phys. Rev. E 60, 1858 (1999).

[15] I. Chuang, B. Yurke, A. N. Pargellis, and N. Turok, Phys. Rev. E 47, 3343 (1993).

[16] D. Melzer and F. R. N. Nabarro, Philos. Mag. 35, 907 (1977).

[17] S.-H. Chen and B. J. Liang, Appl. Phys. Lett. 59, 1173 (1991).

[18] G. De Luca and A. D. Rey, J. Chem. Phys. 127, 104902 (2007).

[19] B.-J. Liang and S.-H. Chen, J. Appl. Phys. 71, 2189 (1992).

[20] A. Rüdinger and H. Stark, Liq. Cryst. 26, 753 (1999).
[21] P. Poulin, H. Stark, T. C. Lubensky, and D. A. Weitz, Science 275, 1770 (1997).

[22] M. J. Press and A. S. Arrott, Phys. Rev. Lett. 33, 403 (1974); J. Phys. Colloques 36, C1-177 (1975).

[23] H. Stark, Eur. Phys. J. B 10, 311 (1999); Phys. Rep. 351, 387 (2001).

[24] R. James and J.-i. Fukuda (unpublished).

[25] J.-i. Fukuda, H. Stark, M. Yoneya, and H. Yokoyama, Phys. Rev. E 69, 041706 (2004).

[26] G. McKay and E. G. Virga, Phys. Rev. E 71, 041702 (2005).

[27] P. M. Morse and H. Feshbach, Methods of Theoretical Physics (McGraw-Hill, New York, 1953).

[28] R. James and J.-i. Fukuda, Phys. Rev. E 88, 010501 (2013).

[29] W. Stein et al., Sage Mathematics Software, Version 5.10, Sage Development Team, University of Washington, 2013.

[30] T. C. Lubensky, D. Pettey, N. Currier, and H. Stark, Phys. Rev. E 57, 610 (1998). 